\title{
B vitamins attenuate the epigenetic effects of ambient fine particles in a pilot human intervention trial
}

\author{
Jia Zhong ${ }^{\mathrm{a}, 1}$, Oskar Karlsson ${ }^{\mathrm{b}, \mathrm{c}}$, Guan Wang ${ }^{\mathrm{d}}$, Jun Li ${ }^{\mathrm{e}, \mathrm{f}}$, Yichen Guog, Xinyi Lin ${ }^{\mathrm{h}}$, Michele Zemplenyi ${ }^{\mathrm{g}}$, \\ Marco Sanchez-Guerra', Letizia Trevisi ${ }^{j}$, Bruce Urch ${ }^{k, 1, m, n}$, Mary Speck ${ }^{k}$, Liming Liang ${ }^{g}$, Brent A. Coull ${ }^{9}$, Petros Koutrakis ${ }^{j}$, \\ Frances Silverman ${ }^{\mathrm{k}, \mathrm{l}, \mathrm{m}, \mathrm{n}}$, Diane R. Gold ${ }^{\mathrm{j}, \mathrm{o}}$, Tangchun $\mathrm{Wu}^{\mathrm{e}, \mathrm{f}}$, and Andrea A. Baccarelli ${ }^{\mathrm{a}}$
}

\begin{abstract}
${ }^{a}$ Mailman School of Public Health, Columbia University, New York, NY 10032; ${ }^{b}$ Center for Molecular Medicine, Department of Clinical Neuroscience, Karolinska Institutet, 17177 Stockholm, Sweden; 'Department of Pharmaceutical Biosciences, Uppsala University, 75237 Uppsala, Sweden; ${ }^{\text {Institute for }}$

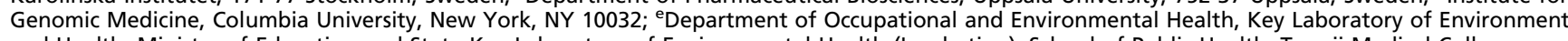
and Health, Ministry of Education and State Key Laboratory of Environmental Health (Incubating), School of Public Health, Tongji Medical College, Huazhong University of Science and Technology, Wuhan, Hubei, China, 430030; 'Department of Epidemiology and Biostatistics, School of Public Health, Tongji Medical College, Huazhong University of Science and Technology, Wuhan, 430030 Hubei, China, 430030; ${ }^{9}$ Department of Biostatistics, T. H. Chan School of Public Health, Harvard University, Boston, MA 02115; 'hingapore Institute for Clinical Sciences, Singapore 117609; i Department of Developmental Neurobiology, National Institute of Perinatology, Mexico City 11000, Mexico; 'Department of Environmental Health, T. H. Chan School of Public Health, Harvard University, Boston, MA 02115; ${ }^{\text {k}}$ Division of Occupational \& Environmental Health, Dalla Lana School of Public Health, University of Toronto, Toronto, ON M5T 3M7, Canada; 'Department of Medicine, University of Toronto, Toronto, ON M5S 1A8, Canada; 'mLi Ka Shing Knowledge Institute, St.

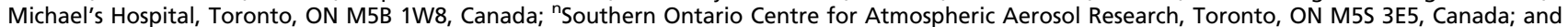
${ }^{\circ}$ Channing Laboratory, Brigham and Women's Hospital, Harvard Medical School, Boston, MA 02115
\end{abstract}

Edited by Kirk R. Smith, University of California, Berkeley, CA, and approved February 13, 2017 (received for review November 8, 2016)

\begin{abstract}
Acute exposure to fine particle $\left(\mathrm{PM}_{2.5}\right)$ induces DNA methylation changes implicated in inflammation and oxidative stress. We conducted a crossover trial to determine whether B-vitamin supplementation averts such changes. Ten healthy adults blindly received a 2-h, controlled-exposure experiment to sham under placebo, $\mathrm{PM}_{2.5}\left(250 \mu \mathrm{g} / \mathrm{m}^{3}\right)$ under placebo, and $\mathrm{PM}_{2.5}\left(250 \mu \mathrm{g} / \mathrm{m}^{3}\right)$ under B-vitamin supplementation ( $2.5 \mathrm{mg} / \mathrm{d}$ folic acid, $50 \mathrm{mg} / \mathrm{d}$ vitamin $B_{6}$, and $1 \mathrm{mg} / \mathrm{d}$ vitamin $B_{12}$ ), respectively. We profiled epigenome-wide methylation before and after each experiment using the Infinium HumanMethylation450 BeadChip in peripheral $\mathrm{CD}^{+}{ }^{+}$T-helper cells. $\mathbf{P M}_{2.5}$ induced methylation changes in genes involved in mitochondrial oxidative energy metabolism. B-vitamin supplementation prevented these changes. Likewise, $\mathbf{P M}_{2.5}$ depleted $11.1 \%$ [95\% confidence interval $(\mathrm{Cl}), 0.4 \%, 21.7 \% ; P=0.04]$ of mitochondrial DNA content compared with sham, and B-vitamin supplementation attenuated the $\mathrm{PM}_{2.5}$ effect by $102 \%\left(P_{\text {interaction }}=0.01\right)$. Our study indicates that individual-level prevention may be used to complement regulations and control potential mechanistic pathways underlying the adverse $\mathrm{PM}_{2.5}$ effects, with possible significant public health benefit in areas with frequent $\mathrm{PM}_{2.5}$ peaks.
\end{abstract}

air pollution | B vitamins | DNA methylation | mitochondria

$\mathbf{H}$ istorical episodes of air pollution peaks were shown to be associated with up to $>10$ times increased death rates (1). According to the WHO, $92 \%$ of the world's population currently lives in places where air quality levels exceed the WHO limits (2). Ambient $\mathrm{PM}_{2.5}$ (particles with an aerodynamic diameter of $<2.5 \mu \mathrm{m}$ ) pollution is one of the most prominent air pollutants (3), because they deposit in the respiratory bronchioles and the alveoli and stimulate local and systemic inflammation and oxidative stress (4). Over the past few decades, substantial lowering of ambient $\mathrm{PM}_{2.5}$ levels has been achieved through large-scale emissions control policies (5). However, exposure peaks with adverse health consequences are still frequently recorded $(6,7)$, even in areas typically exhibiting low levels (5). The molecular mechanisms underlying $\mathrm{PM}_{2.5}$ 's health effects are not fully understood, and the lack of preventative options at the individual level adds complexity to tackling this major public health challenge.

Recent studies in environmental epigenetics provide opportunities to understand the mechanistic underpinnings of exposure-related health effects and to develop novel individual-level interventions. DNA methylation, a potentially modifiable epigenetic mechanism, can regulate gene expression and chromosome integrity via addition of methyl groups to cytosine residues (8). The dynamic DNA landscape can be rapidly altered in peripheral leukocytes following $\mathrm{PM}_{2.5}$ exposure; indeed, such changes are postulated to underlie PMinduced systemic inflammation and oxidative stress $(9,10)$. Most evidence of this phenomenon in humans is based on a heterogeneous mixture of leukocytes (9-12), but a loss of methylation in inflammatory genes and subsequent inflammatory responses, specifically in circulating Th cells, are observed in vivo after environmental challenge $(13,14)$. Notably, DNA methylation is dependent on a biochemical cycle that supplies methyl groups $\left(\mathrm{CH}_{3}\right)$ while relying on methyl nutrients (i.e., $\mathrm{B}$ vitamins including folic acid, vitamin $\mathrm{B}_{6}$ and $\mathrm{B}_{12}$; and amino acids including methionine, betaine, and choline) (8, $15,16)$. In animal studies, a methyl-nutrient-deficient diet leads to aberrant DNA methylation patterns (17), and administration of methyl nutrients enables restoration of epigenetic status (15, 18-20). Likewise, human studies show that dietary methyl nutrient intervention influences the plasticity of DNA methylation (21). The potential for epigenetic modulation has also been demonstrated in the presence of environmental stressors in animal models-Dolinoy et al. successfully used methyl nutrients to avert the DNA hypomethylation induced by bisphenol A exposure (22). These findings

\section{Significance}

Air pollution is a major public health concern worldwide. The molecular mechanistic underpinnings of the health effects of air pollution are not fully understood, and the lack of individuallevel preventative options represent a critical knowledge gap. Our study demonstrated the epigenetic effects of air pollution and suggested that B vitamins might be used as prevention to complement regulations to attenuate the impact of air pollution on the epigenome. Our study inaugurated a line of research for the development of preventive interventions to minimize the adverse effects of air pollution on potential mechanistic markers. Because of the central role of epigenetic modifications in mediating environmental effects, our findings might be extended to other toxicants and environmental diseases.

Author contributions: J.Z., P.K., F.S., D.R.G., and A.A.B. designed research; J.Z., O.K., G.W., B.U., and M.S. performed research; J.Z., O.K., G.W., J.L., X.L., M.Z., M.S.-G., L.T., M.S., L.L., B.A.C., and T.W. contributed new reagents/analytic tools; J.L. and T.W. provided the replication dataset for revision; X.L. advised epigenome-wide analysis; L.T. served as data manager; L.L. and B.A.C. advised biostatistics; P.K. designed the exposure devices; J.Z. and Y.G. analyzed data; and J.Z. wrote the paper.

The authors declare no conflict of interest.

This article is a PNAS Direct Submission.

${ }^{1}$ To whom correspondence should be addressed. Email: jiazhong@mail.harvard.edu.

This article contains supporting information online at www.pnas.org/lookup/suppl/doi:10. 1073/pnas.1618545114/-/DCSupplemental. 
opened new avenues for the application of epigenetic intervention to reduce the health effects of air pollution. However, to date, epigenetic intervention in humans in the context of air pollution has not been described.

The present study is a placebo-controlled crossover pilot intervention trial with controlled human exposure experiments to concentrated ambient fine particles $\left(\mathrm{PM}_{2.5}\right)$. We hypothesized that acute $\mathrm{PM}_{2.5}$ exposure can rapidly modify the DNA methylation profile in peripheral $\mathrm{CD}^{+}$Th cells-the most prolific cytokine producer mediating $\mathrm{PM}_{2.5}$-induced inflammatory responses - and that $\mathrm{PM}_{2.5}$-induced DNA methylation changes can be reduced by $\mathrm{B}$ vitamins (i.e., folic acid, vitamin $\mathrm{B}_{6}$, and $\mathrm{B}_{12}$ ), the primary source of methyl groups (Fig. 1).

\section{Results}

Study Population, Plasma B-Vitamin Concentrations, and Exposure Levels. In the present crossover placebo-controlled trial, 10 volunteers completed 30 controlled exposure experiments following the same order (Fig. S1): seven volunteers aged 19-29 y and three aged 30-49 y. Four volunteers were white, three were Asian, and three were other races. Six volunteers were female and three volunteers had a body mass index $(\mathrm{BMI}) \geq 25$. All volunteers maintained consistent dietary patterns throughout the trial.

The targeted concentration of $\mathrm{PM}_{2.5}$ exposures was $250 \mu \mathrm{g} / \mathrm{m}^{3}$. However, the actual $\mathrm{PM}_{2.5}$ concentrations varied among controlled exposure experiments to $\mathrm{PM}_{2.5}$ (100.6-287.5 $\mu \mathrm{g} / \mathrm{m}^{3}$; median: $\left.234.0 \mu \mathrm{g} / \mathrm{m}^{3}\right)$. There was no significant difference $(P=0.38)$ in $\mathrm{PM}_{2.5}$ concentration between $\mathrm{PM}_{2.5}$ experiment under placebo [median: $219.1 \mu \mathrm{g} / \mathrm{m}^{3}$; interquartile range (IQR): $\left.33.1 \mu \mathrm{g} / \mathrm{m}^{3}\right]$ and $\mathrm{PM}_{2.5}$ experiment under B vitamins (median: $237.2 \mu \mathrm{g} / \mathrm{m}^{3}$; IQR: $48.7 \mu \mathrm{g} / \mathrm{m}^{3}$ ).

To confirm that supplementation affected circulating nutrient levels, we measured plasma B vitamins before and after placebo and supplementation. The median plasma concentrations of folic acid and vitamins $B_{6}$ and $B_{12}$ were $35 \mathrm{nmol} / \mathrm{L}$ (IQR: $14 \mathrm{nmol} / \mathrm{L}$ ), $41 \mathrm{nmol} / \mathrm{L}$ (IQR: $16 \mathrm{nmol} / \mathrm{L}$ ), and $292 \mathrm{pmol} / \mathrm{L}$ (IQR: $72 \mathrm{pmol} / \mathrm{L}$ ) before sham experiment, respectively. After volunteers took placebos for $4 \mathrm{wk}$, their median plasma concentrations were similar: $39 \mathrm{nmol} / \mathrm{L}$ (IQR: $24 \mathrm{nmol} / \mathrm{L})$ for folic acid $(P=0.82), 37 \mathrm{nmol} / \mathrm{L}$ (IQR: $18 \mathrm{nmol} / \mathrm{L})$ for vitamin $\mathrm{B}_{6}(P=0.75)$, and $262 \mathrm{pmol} / \mathrm{L}$ (IQR: $214 \mathrm{pmol} / \mathrm{L})$ for vitamin $\mathrm{B}_{12}(P=0.42)$. B-vitamin supplementation significantly increased the median plasma concentrations of folic acid $(56 \mathrm{nmol} / \mathrm{L}$; IQR: $13 ; P=0.02)$, vitamin $\mathrm{B}_{6}$ (428 nmol/L; IQR: $321 ; P=0.004)$, and vitamin $\mathrm{B}_{12}(511 \mathrm{pmol} / \mathrm{L}$; IQR: $85 ; P=0.01)$. The abovementioned $P$ values were based on Wilcoxon signed-rank test, a nonparametric paired difference test.

CD4 $^{+}$Th Cell Purity. All CD4 ${ }^{+}$Th cell samples' purity were over $80 \%$ (Table S1), with only minor contamination from CD8
T cells, B cells, granulocytes, and natural killer cells. The median purity of samples collected at sham experiment, $\mathrm{PM}_{2.5}$ experiment under placebo, and $\mathrm{PM}_{2.5}$ experiment under $\mathrm{B}$ vitamins was $96.9 \%$ (IQR: 5.1), 94.8\% (IQR: 7.1), and 96.1\% (IQR: 4.7), respectively.

Effect of $\mathbf{P M}_{2.5}$ and B-Vitamin Supplementation on DNA Methylation. Two-hour $\mathrm{PM}_{2.5}$ exposure substantially modified DNA methylation in $\mathrm{CD}^{+}{ }^{+}$Th cells, and these changes were prevented by Bvitamin supplementation (Fig. 2). Because our study is limited in power, we present only the top 10 loci, selected following the method proposed by Maccani et al. (23) —first based on effect size and then $P$ value (Table S2 and Fig. $2 A$ ). In the absence of B-vitamin supplementation, $\mathrm{PM}_{2.5}$ exposure either increased or decreased DNA methylation levels, compared with sham, at these loci (Fig. 2A). Quantile-Quantile plots for expected vs. observed distribution of $P$ values showed minimal genomic inflation with a lambda of 1.03 (Fig. $2 B$ ).

Table S2 presents the genomic position, relation to $\mathrm{CpG}$ islands, and gene symbol for the 10 loci. The top two loci that are associated with known genes were cg06194186 and cg17157498. Locus cg06194186 is located in the promoter region (TSS1500) of the carboxypeptidase $\mathrm{O}(C P O)$ gene, and locus cg17157498 is located in the promoter region (TSS1500) of the NADH dehydrogenase (ubiquinone) Fe-S protein 7 (NDUFS7) gene (Figs. S2 and S3). Fig. 3 presents the shift in methylation level distributions and corresponding IQRs pre and post each exposure experiment for these two loci.

Four-week B-vitamin supplementation attenuated the $\mathrm{PM}_{2.5}$ effect by $28-76 \%$ at the top 10 loci. Supplementing B vitamins resulted in a reduction in effect size by $57 \%$ for $\operatorname{cg} 06194186$, $49 \%$ for $\operatorname{cg} 07689821,73 \%$ for $\operatorname{cg} 00068102,31 \%$ for $\operatorname{cg} 00647528$, $45 \%$ for $\operatorname{cg} 15426626,28 \%$ for $\operatorname{cg} 10719920,76 \%$ for $\operatorname{cg} 21986027$, $74 \%$ for $\operatorname{cg} 17157498$, $63 \%$ for $\operatorname{cg} 08075528$, and $71 \%$ for cg26995744, respectively (Table S3 and Fig. 2C).

Effect of $\mathbf{P M}_{2.5}$ and B-Vitamin Supplementation on Mitochondrial DNA Content. $C P O$ and NDUFS7 are both involved in mitochondrial oxidative energy metabolism - a pivotal function with substantial impact on mitochondrial biogenesis and clearance $(24,25)$. In the secondary exploratory analysis, we further tested the associations of $\mathrm{PM}_{2.5}$ with mitochondrial DNA content, as well as the potential protective effect of B-vitamin supplementation. In the absence of B-vitamin supplementation, compared with sham, 2-h exposure to $\mathrm{PM}_{2.5}$ was estimated to be nonsignificantly associated with a $-0.3 \%$ change [95\% confidence interval (CI): $-10.1 \%, 9.5 \% ; P=0.94]$ in mitochondrial DNA content. However, $24 \mathrm{~h}$ after exposure experiments, we observed substantial reduction in mitochondrial DNA content associated with $\mathrm{PM}_{2.5}$ : 2-h exposure to $\mathrm{PM}_{2.5}$ significantly depleted mitochondrial DNA content by $11.1 \%$ (95\% CI: $0.4 \%$,

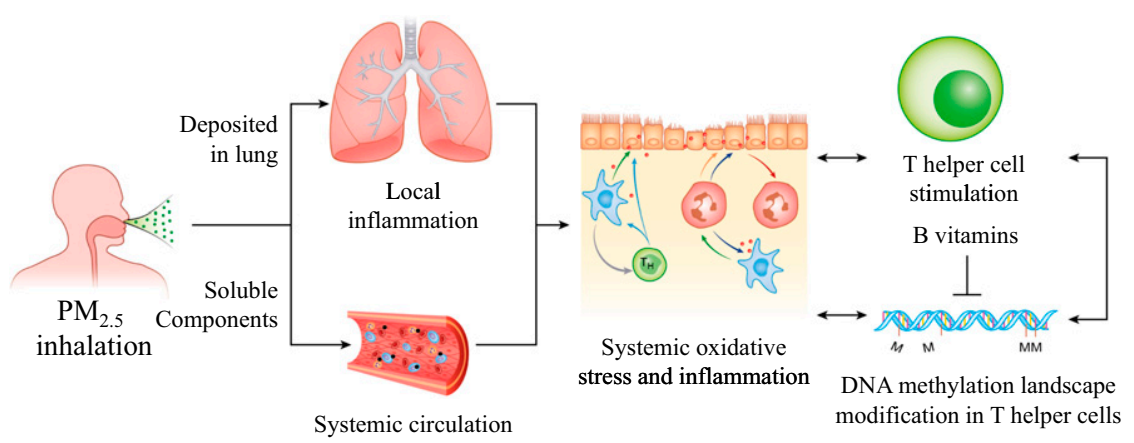

Fig. 1. Proposed conceptual model linking fine particulate matter $\left(P_{2.5}\right)$, systemic oxidative stress and inflammation, and altered DNA methylation landscape in Th cells. We hypothesized that $\mathrm{PM}_{2.5}$ inhalation triggers local and systemic inflammation and oxidative stress, which alters the DNA methylation landscape in circulating $C D 4^{+}$Th cells and further stimulates $C D 4^{+}$Th cells. In return, stimulated CD4 ${ }^{+}$Th cells undergo more epigenetic remodeling-possibly due to modulated methyl group availability-therefore creating a vicious circle which amplifies the inflammatory and oxidative effects of $\mathrm{PM}_{2.5}$. 

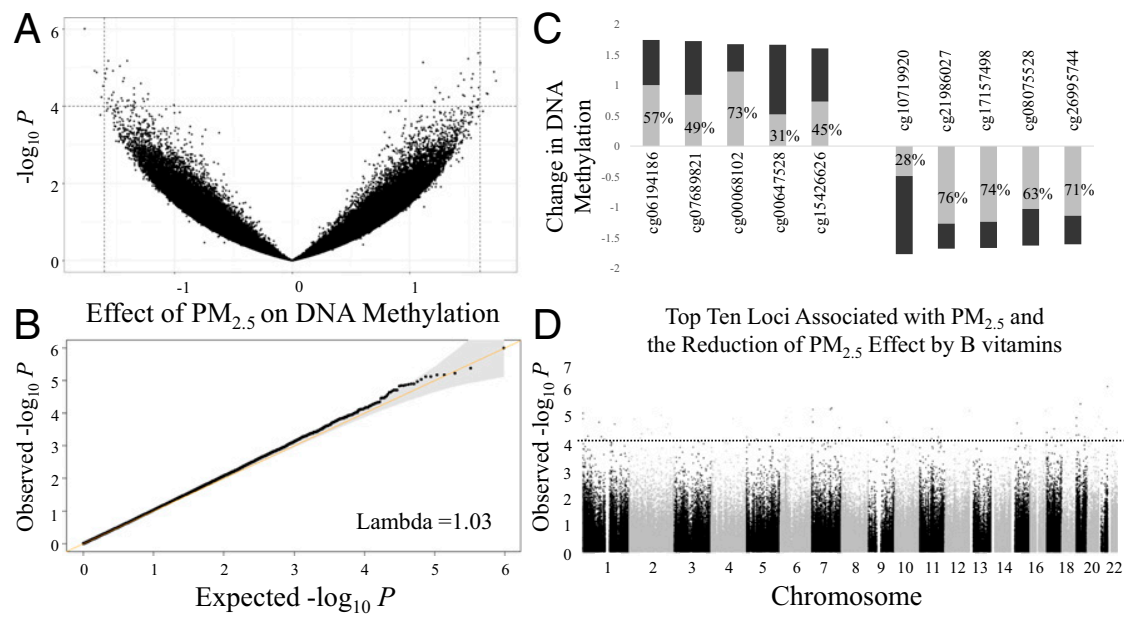

Fig. 2. The effect of 2-h exposure to $\mathrm{PM}_{2.5}$ on DNA methylation landscape and the reduction of $\mathrm{PM}_{2.5}$ effect by $\mathrm{B}$-vitamin supplementation. $A$ is the Volcano plot depicting the distribution of estimated effect of $\mathrm{PM}_{2.5}$ exposure on the epigenome. Each dot represents the estimated PM 2.5 effect for one $\mathrm{CpG}$. The vertical lines indicate suggestive threshold based on effect size, whereas the horizontal line reflects the suggestive threshold based on statistical significance. $B$ is the quantile-quantile plot for associations of $\mathrm{PM}_{2.5}$ with DNA methylation in circulating $\mathrm{CD}^{+}{ }^{+}$Th cells. $C$ represents the top 10 loci associated with $\mathrm{PM}_{2.5}$ and the reduction of $\mathrm{PM}_{2.5}$ effect by $\mathrm{B}$ vitamins. Bar height indicates $\mathrm{PM}_{2.5}$ effect, whereas the gray part indicates the magnitude of effect attenuation by $\mathrm{B}$ vitamins. $D$ is the Manhattan plot representing the chromosome location of each loci. The dashed horizontal line reflects the suggestive threshold for statistical significance. Analyses were adjusted for season, chamber humidity, and temperature.

$21.7 \% ; P=0.04)$ (Table S3). B-vitamin supplementation completely attenuated such effect of $\mathrm{PM}_{2.5}$ by $102 \%\left(P_{\text {interaction }}=0.01\right)$. With B-vitamin supplementation, 2-h exposure to $\mathrm{PM}_{2.5}$ was not associated with mitochondrial DNA content $(0.2 \%$; $95 \%$ CI: $-8.3 \%$, $8.8 \% ; P=0.96)$ (Table S3).

Exploratory Mediation Analysis and External Supporting Data. We further deconstructed total $\mathrm{PM}_{2.5}$ effects on mitochondrial DNA content into direct and indirect (i.e., mediated) effects to investigate whether DNA methylation levels at loci cg06194186 and cg17157498 mediate $\mathrm{PM}_{2.5}$-mitochondrial DNA content relationship. Our result indicated that $16.0 \%$ (95\% CI: $4.1 \%$, $27.9 \%$ ) and $18.4 \%$ (95\% CI: $9.9 \%, 26.9 \%)$ of the PM effect on mitochondrial DNA content was mediated by $\operatorname{cg} 06194186$ and cg17157498 methylation, respectively. Consistent with our hypothesis, we observed correlation between mitochondrial DNA content and the methylation levels of $\operatorname{cg} 06194186(r=-0.45 ; P=$
$0.06)$ and $\operatorname{cg} 17157498(r=0.63 ; P=0.01)$ in $\mathrm{CD}^{+}$Th cells, in an independent external dataset.

Sensitivity Analysis. B vitamins have a long biological half-life (26), therefore requires a washout period longer than four months. We designed the trial without randomizing the treatment order (placebo vs. B vitamins) to avoid long washout periods, which would have made exposure experiments on the same volunteer less comparable. Lifestyle factors may vary over several months, particularly in relation to seasonality, which also may directly affect DNA methylation $(8,10)$. To rule out the potential impact of temporal trend on our results, we adjusted for date-since-entry, and this adjustment did not affect our conclusion. Furthermore, we conducted permutation test on the two top loci to ensure the robustness of our analysis $\left(P_{\text {permulation }}<0.001\right)$. In the analysis involving the mitochondrial DNA content, we additionally adjusted for age (continuous), BMI (continuous), and race (categorical) to examine if our results are
Exposure 1: Sham + Placebo

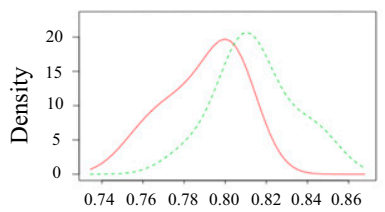

cg06194186 methylation level

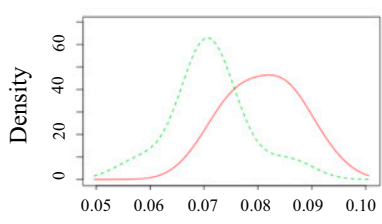

cg17157498 methylation level
Exposure 2: $\mathrm{PM}_{2.5}+$ Placebo

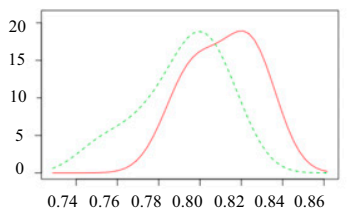

cg06194186 methylation level

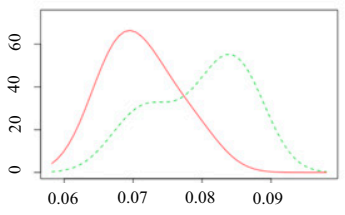

cg17157498 methylation level

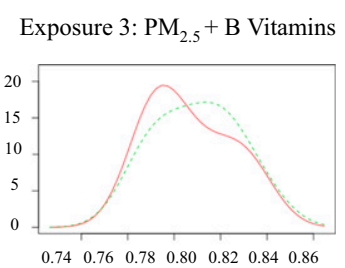

cg06194186 methylation level

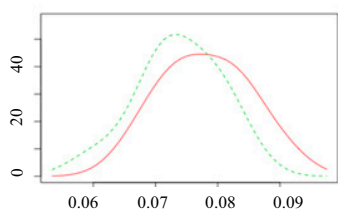

cg17157498 methylation level

$$
\text { Pre Exposure Post Exposure }
$$

Fig. 3. Distribution of DNA methylation levels of cg06194186 and cg17157498 before and after each exposure experiment. Red solid lines and green dashed lines indicate the distribution of methylation levels measured before and after exposure, respectively. 
sensitive to covariates specification. Our results were robust and consistent (Table S3).

\section{Discussion}

This crossover intervention trial with controlled exposure experiments demonstrated that 2-h exposure to concentrated ambient $\mathrm{PM}_{2.5}\left(250 \mu \mathrm{g} / \mathrm{m}^{3}\right)$ affects the dynamic epigenetic landscape in circulating $\mathrm{CD}^{+}$Th cells among healthy adults. We showed that these effects can be prevented with B-vitamin supplementation (i.e., folic acid and vitamins $\mathrm{B}_{6}$ and $\mathrm{B}_{12}$ ). Furthermore-as the top loci suggested potential modulation of mitochondrial metabolism -we followed up these findings by showing that exposure to $\mathrm{PM}_{2.5}$ significantly altered mitochondrial DNA content in circulating $\mathrm{CD}^{+} \mathrm{Th}$ cells, and B-vitamin supplementation nearly completely prevented these effects.

Air pollution has been consistently associated with adverse health outcomes in epidemiological studies $(1,3,4,9)$. Although the biological mechanism underlying the health effects of $\mathrm{PM}_{2.5}$ remains not fully understood, systemic inflammation and oxidative stress have been proposed as essential biological pathways $(4,27)$. Furthermore, $\mathrm{PM}_{2.5}$ can disturb DNA methylation profiles $(9,10,13)$, which might exacerbate oxidative and inflammatory responses following exposure. A previous human exposure study demonstrated that Toll-like receptor 4 (TLR4) gene hypomethylation in leukocytes mediates a part of PM effect on blood pressure (28). Recently, an epigenome-wide association study (EWAS) showed that lowconcentration air pollution alters DNA methylation profiles in whole blood (9). However, the interpretation of those findings is limited, considering potential bias due to cell-type heterogeneity within whole blood, and by the correlational nature of observational studies $(11,12)$. In addition, these findings might not be generalizable to areas with frequent air pollution peaks. The present study-a cell type-specific EWAS using controlled exposure experiments-has the unique advantage of providing unbiased insight on the novel epigenetic underpinnings of the proinflammatory and prooxidative effects of $\mathrm{PM}_{2.5}$ exposure peaks.

In line with our hypothesis, we demonstrated acute effects of $\mathrm{PM}_{2.5}$ inhalation on DNA methylation in the promoter region of genes related to mitochondrial function and oxidative metabolism (24, 25): $C P O$, a member of the metalloenzyme family (25), is involved in metal ion binding, metallopeptidase, and metallocarboxypeptidase activities-which are essential in regulation of the steady-state concentration of $\mathrm{O}_{2}{ }^{-}$in the intermembrane space of mitochondria (29); NDUFS7 encodes one of the subunits of the mitochondrial respiratory chain complex $I$ that transfers electrons from NADH to coenzyme Q, and NDUFS7 mutations were of etiological significance in mitochondrial complex I deficiency (30). Although mitochondria have their own genetic material distinct from the nuclear DNA, the majority of mitochondrial proteins are encoded by the nuclear genome (31). The observation that $\mathrm{PM}_{2.5}$ exposure substantially altered DNA methylation of nuclear genes in mitochondrial pathways indicate that mitochondria-the specialized organelles that regulate cellular-redox-balance and supplies energy-are a primary target of PM-induced cellular responses (32).

Our results on mitochondrial DNA content supplemented the findings from the epigenome-wide DNA methylation scan: exposure to $\mathrm{PM}_{2.5}$ for $2 \mathrm{~h}$ was followed by reduced mitochondrial DNA content 24-h postexposure. The cellular mitochondrial genomic content is stringently regulated by biogenesis/degradation machinery (33), which is vital in the determination of cell survival and function. Compensatory mitochondria biogenesis can buffer an intracellular reactive oxygen species (ROS) challenge, as an adaptive stress response to eliminate cellular oxidative damage (34). However, persistent oxidative stress may eventually overwhelm the adaptive response system and lead to mitochondrial DNA depletion via mitophagy (35). Our results support this hypothesis by demonstrating that exposure to high- concentration $\mathrm{PM}_{2.5}$ can reduce the mitochondrial DNA contents in circulating $\mathrm{CD} 4^{+}$Th cells. Consistent with our results, a recent study reported that a $10 \mu \mathrm{g} / \mathrm{m}^{3}$ increase in coarse PM $\left(\mathrm{PM}_{10-2.5}\right)$ exposure during pregnancy was associated with a $16.1 \%$ decrease in placental mitochondrial DNA content (36). Our exploratory mediation analysis indicates that short-term exposure to high-concentration $\mathrm{PM}_{2.5}$ depletes mitochondrial DNA content, likely via - at least in part-modulating DNA methylation levels of genes in mitochondrial pathways. Future studies are warranted to investigate the potential for targeted epigenetic interventions.

DNA methylation is a modifiable biochemical process relying on methyl-group supplying nutrients such as B vitamins, which is postulated to increase DNA methylation levels $(8,15,16)$. This feature renders B-vitamin supplementation an attractive pharmaceutical intervention to counteract the PM effects, which has been associated with loss of DNA methylation on inflammatory genes (28). Landmark experiments on the Agouti $\mathrm{A}^{\mathrm{vy}}$ mice and other models have shown that dietary methyl nutrients, added during gestation (15-18) or later even in adult life $(20,21)$, can be used to modulate DNA methylation status. In human studies, intake of a folic acid-depleted diet for several weeks promotes hypomethylation of lymphocyte genomic DNA among postmenopausal women, and this hypomethylation is reversible with folic acid replacement (21). Among patients with colorectal adenomatous polyps, folic acid supplementation led to a $31 \%$ increase in leukocyte DNA methylation and a $25 \%$ increase in DNA from the colonic mucosa (37). Potential for human translation is also demonstrated in animal models, as methyl group-supplying nutrients can be used to prevent the loss of DNA methylation induced by environmental pollutants in rodents (22). However, to the best of our knowledge, whether B vitamins can be used to limit adverse effects from PM pollution has not previously been tested in human. Our research provided the experimental evidence showing that the epigenetic effects of $\mathrm{PM}_{2.5}$ can be reduced using 4-wk B-vitamin supplementation. Remarkably, in our data, the B-vitamin supplementation not only prevented decreased DNA methylation but also increased DNA methylation following acute exposure to high-concentration PM. These findings suggest that B vitamins might protect against DNA hypomethylation as methyl group-supplying nutrients. On the other hand, B vitamins might also minimize DNA hypermethylation through interactions with regulatory pathways mediated by essential enzymes (such as DNA methyltransferases and methylenetetrahydrofolate reductase).

A major innovation of the present study over previous human epigenome-wide studies is the use of isolated CD4 ${ }^{+}$Th cells-an essential cell type modulating human immunity through both its own immune activities and regulation of other leukocytes' proliferation, apoptosis, migration, and other functions via cytokine signaling (31). Therefore, the epigenetic effects of $\mathrm{PM}_{2.5}$ and protective effects of $\mathrm{B}$ vitamins observed in $\mathrm{CD}^{+}{ }^{+}$Th genome might indicate subsequent modulation of essential cellular functions of other blood cell types. The Houseman cell proportion estimates indicated high purity of the analyzed samples. Although our study is subject to residual influence from differential CD $4^{+}$Th subsets, the observed effect of $\mathrm{PM}_{2.5}$ or $\mathrm{B}$ vitamins on DNA methylation is unlikely to be surrogate for leukocyte composition variation. Our plating scheme for DNA methylation analysis is independent of the exposure and treatment status, and was designed to minimize potential bias due to technical variables. Thus, the measurement error of DNA methylation can be assumed to be nondifferential and, therefore, likely to bias the results toward null. We conducted sensitivity analysis to robustly evaluate the impact of $\mathrm{PM}_{2.5}$ on top loci, and further supported the EWAS results with a widely accepted mitochondrial marker-mitochondrial DNA content-with a highly reproducible quantitative real-time PCR method. Our 
crossover design controlled for time-invariant factors such as sex, race, BMI, SNP, etc. In addition, all exposure experiments were conducted at the same time of the day to eliminate any impact due to diurnal variation.

We acknowledge several limitations in the present pilot study. Although our EWAS is limited in power to meet the stringent Bonferroni threshold for significance with only 10 volunteers (30 exposure experiments), our sample size is comparable to previous controlled exposure studies that succeeded in demonstrating health effects of PM exposure (28, 38-40). As suggested by previous study (23), we selected the top loci based on both effect size and statistical significance, because those loci are more likely to infer biological significance. The short study duration was implemented to reduce the impact of with-in volunteer seasonal variations. Therefore, we could not randomize on the treatment (placebo vs. B vitamins) order due to long biological half-life of body stores of B vitamins (26), which might create potential confounding due to a temporal trend or learning effect (i.e., the volunteers might be more tolerant with the $\mathrm{PM}_{2.5}$ effects at the second $\mathrm{PM}_{2.5}$ exposure). In the sensitivity analysis, we adjusted for the amount of time passing because the study entry, and our results were consistent. Although residual confounding is possible, considering the magnitude of our effect estimates and the consistency of our findings, it is unlikely that the observed association reflected bias resulting from confounding. In addition, actual $\mathrm{PM}_{2.5}$ concentration in $\mathrm{PM}_{2.5}$ experiments under placebo was nonsignificantly lower than $\mathrm{PM}_{2.5}$ experiments under B-vitamin supplementation, which might bias our results on B vitamins' protective effects toward the null. Finally, future validation studies are warranted because our unique study design using $\mathrm{CD} 4^{+}$Th cells created major challenge to identify a suitable replication cohort, and our findings might not be generalizable to other cell types due to cell-type specificity of DNA methylation and mitochondrial DNA content.

The unclear molecular mechanistic underpinning of $\mathrm{PM}_{2.5}$ 's health effects remains the major gap in current knowledge-thereby creating challenges in developing preventative strategies. The present study is a pilot intervention trial in the investigation of mechanistic pathways underlying the adverse health effects of air pollution, and potential targeted preventive approaches. We demonstrated that ambient $\mathrm{PM}_{2.5}$ exposure peak has unfavorable effects on epigenetic and pro-oxidative markers that can be neutralized by B-vitamin supplementation. Our findings suggest promising opportunities to aid the development of novel intervention strategieswhich is particularly important for pathologies related to ubiquitous exposures such as $\mathrm{PM}_{2.5}$ pollution. Future trials with larger sample sizes are warranted to shed light on the precise pathophysiological processes of PM-induced inflammatory and oxidative responses, the mechanism underlying the protective effect of B vitamins, and potential clinical application.

\section{Methods}

Study Population. We recruited 10 healthy, 18- to 60-y-old, nonsmoking volunteers who were not taking any medicines or vitamin supplements, from the University of Toronto campus and surrounding area. The trial protocols were approved by all participating institutional review board (University of Toronto, St. Michael's Hospital, and Harvard T.H. Chan School of Public Health) and registered (ClinicalTrials.gov identifier NCT01864824; date of registration: May 8, 2013). All methods were performed in accordance with the relevant guidelines and regulations. We obtained written informed consent from every volunteer before enrollment.

Study Design. We conducted a single-blind, crossover intervention trial with controlled exposure experiments to concentrated ambient $\mathrm{PM}_{2.5}$ (July 2013 to February 2014). The design (Fig. 1) started with a 2-wk run-in period with placebo, followed by the baseline sham experiment $(2 \mathrm{~h}$, particle-free medical air, exposure one). After sham experiment, each volunteer took placebo for $4 \mathrm{wk}$ and was then exposed to $\mathrm{PM}_{2.5}(2 \mathrm{~h}$, target concentration: $250 \mu \mathrm{g} / \mathrm{m}^{3}$, exposure two). Volunteers started the 4-wk B-vitamin supplementation after exposure two, and then were exposed again to $\mathrm{PM}_{2.5}$ ( $2 \mathrm{~h}$, target concentration: $250 \mu \mathrm{g} / \mathrm{m}^{3}$, exposure three). All volunteers received three exposure experiments following the same order.

Exposure Facility. Ambient particles were drawn in from an inlet $1.5 \mathrm{~m}$ high, beside a busy ( $>1,000$ vehicles per hour) street in downtown Toronto. We used the Harvard ambient particle concentrator to generate concentrated ambient $\mathrm{PM}_{2.5}$ (41), and delivered $\mathrm{PM}_{2.5}$ air stream to the volunteer seated inside a $4.9-\mathrm{m}^{3}(1.1 \times 1.9 \times 2.0 \mathrm{~m})$ lexan enclosure via an "oxygen type" facemask. The sham exposure with medical air were generated as previously described (38). During each exposure experiment, $\mathrm{PM}_{2.5}$ mass was collected on a 47-mm, 2- $\mu \mathrm{m}$ Teflon filter (Teflo, R2PJ047; Pall Corp.) and was monitored using the gravimetric determination of $\mathrm{PM}_{2.5}$ exposure mass concentration (micrograms per cubic meter).

Folic Acid, Vitamin $\mathbf{B}_{\mathbf{6}}$, and Vitamin $\mathbf{B}_{\mathbf{1 2}}$ Supplement. We administered one placebo or B-vitamin supplement $\left(2.5 \mathrm{mg}\right.$ of folic acid, $50 \mathrm{mg}$ of vitamin $\mathrm{B}_{6}$, and $1 \mathrm{mg}$ of vitamin $B_{12}$ ) daily to each volunteer. Previous human trials showed that these doses rapidly increased plasma B-vitamin levels, modified methyl-cycle metabolite levels, and ameliorated cardiovascular measurements $(42,43)$. Preparation, packaging, and coding of the placebo and supplement were done by an external laboratory (Jamieson Laboratories) and was blinded to the volunteers. Before each exposure experiment, we measured volunteers' plasma folic acid and vitamin $B_{12}$ levels using competitive-binding immunoenzymatic assay (A98032 and 33000; Beckman Coulter), and vitamin $B_{12}$ levels using HPLC with fluorescence detection. At the first and the last visit, we assessed typical daily B-vitamin intake with a self-administered validated (44), semiquantitative food-frequency questionnaire used in the Nurses' Health Study.

CD4 $^{+}$Th Cell Isolation and DNA Extraction. We collected blood samples via venous phlebotomy (preexposure, immediately postexposure, and $24 \mathrm{~h}$ postexposure), and within $4 \mathrm{~h}$, isolated $\mathrm{CD} 4^{+}$Th cells by removing unwanted cells using RosetteSep Human CD4 ${ }^{+}$T Cell Enrichment Mixture (no. 15062; Stem Cell Technologies). DNA was then extracted using a Promega Maxwell 16 instrument with tissue DNA purification kit (Promega). We monitored the concentration and quality of extracted DNA using NanoDrop ND-1000 spectrophotometer (Nanodrop Technologies). Unsatisfactory DNA samples were discarded and DNA was extracted again. We estimated the proportions of major leukocyte types (CD4 T cells, CD8 T cells, B cells, granulocytes, monocytes, and natural killer cells) to assess the purity of isolated CD4 ${ }^{+}$Th cells using the Houseman method, a statistical deconvolution technique based on the $450 \mathrm{~K}$ data (11).

Epigenome-Wide DNA Methylation Scan. We measured the epigenome-wide DNA methylation profile using the Infinium Human Methylation 450K BeadChip (Illumina) (45), which allows the assessment of approximately half a million CpG sites across $99 \%$ of RefSeq genes within the genome (46). Because of the within-volunteer, cross-over design, we plated all samples from one volunteer in one chip, with pre- and postexposure samples randomly loaded onto each column on the same row. All samples were processed by one technician and analyzed in one batch to minimize batch effect. Sample preparation and quality control details are explained in SI Methods.

Mitochondrial DNA Content in CD4 ${ }^{+}$Th Cells. We measured mitochondrial DNA content in $\mathrm{CD}^{+}$Th cells through the mtDNA/nDNA ratio, a widely used biomarker representing the mitochondrial DNA copy number versus the nuclear DNA copy number (34). Mitochondrial DNA copy number was analyzed pre, immediately after, and $24 \mathrm{~h}$ after each exposure experiment using multiplex quantitative real-time $P C R$, as previously reported (34). The mtDNA/nDNA is used in the statistical analysis - a ratio value of 1 indicates that the mtDNA/nDNA of the test sample is equal to the mtDNA/nDNA in the reference DNA pool used in the assay.

CD4 $^{+}$Th Mitochondrial DNA Content and DNA Methylation in External Dataset. To strengthen our findings, we identified an external dataset based on 15 deidentified volunteers' $\mathrm{CD}^{+} \mathrm{T}$ cells-which were purified from fresh blood samples through magnetic-activated cell sorting using anti-CD4 antibody coupled paramagnetic microbeads (Miltenyi Biotec). Epigenome-wide DNA methylation profiles and mitochondrial DNA content were measured using the same methods of the present study. 
Statistical Methods. We used linear mixed-effects models (SI Methods) with random intercepts assigned to each volunteer to account for correlation among within-volunteer measurements. The crossover design minimized the influence from time-invariant factors. In all models, we adjusted for time-varying covariates with potential influences on DNA methylation, selected based on prior knowledge and the existing literature [i.e., season (fall/winter/spring/summer) chamber temperature, and chamber relative humidity (38)]. Rank-based normal transformation was performed on all DNA methylation measures to improve normality and stabilize the variance. We further performed permutation tests on the observed top two loci to ensure the robustness of our results, and conducted exploratory mediation analysis to evaluate whether DNA methylation mediates

1. Bell ML, Davis DL (2001) Reassessment of the lethal London fog of 1952: Novel indicators of acute and chronic consequences of acute exposure to air pollution. Environ Health Perspect 109(Suppl 3):389-394.

2. World Health Organization (September 27, 2016) WHO releases country estimates on air pollution exposure and health impact. Press release. Available at www.who. int/mediacentre/news/releases/2016/air-pollution-estimates/en/. Accessed February 24, 2017.

3. Lelieveld J, Evans JS, Fnais M, Giannadaki D, Pozzer A (2015) The contribution of outdoor air pollution sources to premature mortality on a global scale. Nature 525(7569):367-371.

4. Brook RD, et al.; American Heart Association Council on Epidemiology and Prevention, Council on the Kidney in Cardiovascular Disease, and Council on Nutrition, Physical Activity and Metabolism (2010) Particulate matter air pollution and cardiovascular disease: An update to the scientific statement from the American Heart Association. Circulation 121(21):2331-2378.

5. World Health Organization (2006) Air Quality Guidelines: Global Update 2005 Particulate Matter, Ozone, Nitrogen Dioxide, and Sulfur Dioxide (World Health Organization, Copenhagen).

6. Richmond-Bryant J, Saganich C, Bukiewicz L, Kalin R (2009) Associations of PM 2.5 and black carbon concentrations with traffic, idling, background pollution, and meteorology during school dismissals. Sci Total Environ 407(10):3357-3364.

7. Bender A, et al. (2009) Understanding Episodes of High Airborne Particulate Matter in lowa (Bistate Regional Commission in Eastern lowa, lowa City, IA).

8. Ramchandani S, Bhattacharya SK, Cervoni N, Szyf M (1999) DNA methylation is a reversible biological signal. Proc Natl Acad Sci USA 96(11):6107-6112.

9. Panni T, et al. (2016) A genome-wide analysis of DNA methylation and fine particulate matter air pollution in three study populations: KORA F3, KORA F4, and the Normative Aging Study. Environ Health Perspect 124(7):983-90.

10. Baccarelli A, et al. (2009) Rapid DNA methylation changes after exposure to traffic particles. Am J Respir Crit Care Med 179(7):572-578.

11. Houseman EA, et al. (2012) DNA methylation arrays as surrogate measures of cell mixture distribution. BMC Bioinformatics 13:86

12. Rakyan VK, Down TA, Balding DJ, Beck S (2011) Epigenome-wide association studies for common human diseases. Nat Rev Genet 12(8):529-541.

13. Liu J, et al. (2008) Combined inhaled diesel exhaust particles and allergen exposure alter methylation of T helper genes and IgE production in vivo. Toxicol Sci 102(1):76-81.

14. Bruniquel D, Schwartz RH (2003) Selective, stable demethylation of the interleukin-2 gene enhances transcription by an active process. Nat Immunol 4(3):235-240.

15. Cropley JE, Suter CM, Martin DI (2007) Methyl donors change the germline epigenetic state of the $A(v y)$ allele. FASEB J 21(12):3021, author reply 3021-3022.

16. Dolinoy DC, Weidman JR, Waterland RA, Jirtle RL (2006) Maternal genistein alters coat color and protects Avy mouse offspring from obesity by modifying the fetal epigenome. Environ Health Perspect 114(4):567-572.

17. Sinclair KD, et al. (2007) DNA methylation, insulin resistance, and blood pressure in offspring determined by maternal periconceptional $B$ vitamin and methionine status. Proc Natl Acad Sci USA 104(49):19351-19356.

18. Waterland RA, et al. (2006) Maternal methyl supplements increase offspring DNA methylation at Axin Fused. Genesis 44(9):401-406.

19. Weaver IC, et al. (2005) Reversal of maternal programming of stress responses in adult offspring through methyl supplementation: Altering epigenetic marking later in life. J Neurosci 25(47):11045-11054.

20. Kotsopoulos J, Sohn KJ, Kim YI (2008) Postweaning dietary folate deficiency provided through childhood to puberty permanently increases genomic DNA methylation in adult rat liver. J Nutr 138(4):703-709.

21. Jacob RA, et al. (1998) Moderate folate depletion increases plasma homocysteine and decreases lymphocyte DNA methylation in postmenopausal women. J Nutr 128(7): 1204-1212.

22. Dolinoy DC, Huang D, Jirtle RL (2007) Maternal nutrient supplementation counteracts bisphenol A-induced DNA hypomethylation in early development. Proc Natl Acad SC USA 104(32):13056-13061.

23. Maccani JZ, et al. (2015) Placental DNA methylation related to both infant toenail mercury and adverse neurobehavioral outcomes. Environ Health Perspect 123(7):723-729.

24. Hyslop SJ, Duncan AM, Pitkänen S, Robinson BH (1996) Assignment of the PSST subunit gene of human mitochondrial complex I to chromosome 19p13. Genomics 37(3):375-380. the effect of $\mathrm{PM}_{2.5}$ on mitochondrial DNA content (SI Methods). Analyses were performed using SAS 9.4 (SAS Institute) and R statistical computing software (R Foundation for Statistical Computing).

Data Availability. Data are available on request due to privacy or other restrictions.

ACKNOWLEDGMENTS. This study was supported by NIH Grants R21ES021895 R01ES015172, R01ES021733, R01ES020836, R01ES021357, P30ES009089, and P30ES000002; US Environmental Protection Agency funding (Grant RD83479801); Environment Canada; AllerGen Networks of Centres of Excellence and National Natural Science Foundation of China Grant 91643202.

25. Wei S, et al. (2002) Identification and characterization of three members of the human metallocarboxypeptidase gene family. J Biol Chem 277(17):14954-14964.

26. Butler $C C$, et al. (2006) Oral vitamin $B_{12}$ versus intramuscular vitamin $B_{12}$ for vitamin $B_{12}$ deficiency: A systematic review of randomized controlled trials. Fam Pract 23(3): 279-285.

27. Ghio AJ, Carraway MS, Madden MC (2012) Composition of air pollution particles and oxidative stress in cells, tissues, and living systems. J Toxicol Environ Health B Crit Rev 15(1):1-21.

28. Bellavia A, et al. (2013) DNA hypomethylation, ambient particulate matter, and increased blood pressure: Findings from controlled human exposure experiments. J Am Heart Assoc 2(3):e000212.

29. Turrens JF (2003) Mitochondrial formation of reactive oxygen species. J Physio/ 552(Pt 2):335-344.

30. Smeitink J, van den Heuvel L (1999) Human mitochondrial complex I in health and disease. Am J Hum Genet 64(6):1505-1510.

31. Cooper G (2000) The Cell: A Molecular Approach (Sinauer Associates, Sunderland, MA), 2nd Ed.

32. Lee HC, Wei YH (2005) Mitochondrial biogenesis and mitochondrial DNA maintenance of mammalian cells under oxidative stress. Int J Biochem Cell Biol 37(4): 822-834.

33. Attardi G, Schatz G (1988) Biogenesis of mitochondria. Annu Rev Cell Bio/ 4:289-333.

34. Zhong J, et al. (2015) Traffic-related air pollution, blood pressure, and adaptive response of mitochondrial abundance. Circulation 133(4):378-87.

35. Kim I, Rodriguez-Enriquez S, Lemasters JJ (2007) Selective degradation of mitochondria by mitophagy. Arch Biochem Biophys 462(2):245-253.

36. Janssen BG, et al. (2012) Placental mitochondrial DNA content and particulate air pollution during in utero life. Environ Health Perspect 120(9):1346-1352.

37. Pufulete $M$, et al. (2005) Effect of folic acid supplementation on genomic DNA methylation in patients with colorectal adenoma. Gut 54(5):648-653

38. Behbod B, et al. (2013) Endotoxin in concentrated coarse and fine ambient particles induces acute systemic inflammation in controlled human exposures. Occup Environ Med 70(11):761-767.

39. Brook RD, et al. (2002) Inhalation of fine particulate air pollution and ozone causes acute arterial vasoconstriction in healthy adults. Circulation 105(13):1534-1536.

40. Urch $B$, et al. (2004) Relative contributions of $P_{2.5}$ chemical constituents to acute arterial vasoconstriction in humans. Inhal Toxicol 16(6-7):345-352.

41. Demokritou P, Gupta T, Ferguson S, Koutrakis P (2003) Development of a highvolume concentrated ambient particles system (CAPS) for human and animal in halation toxicological studies. Inhal Toxicol 15(2):111-129.

42. Lonn E, et al.; Heart Outcomes Prevention Evaluation (HOPE) 2 Investigators (2006) Homocysteine lowering with folic acid and B vitamins in vascular disease. $N$ Engl $J$ Med 354(15):1567-1577.

43. Ullegaddi R, Powers HJ, Gariballa SE (2004) B-group vitamin supplementation mitigates oxidative damage after acute ischaemic stroke. Clin Sci (Lond) 107(5):477-484

44. Willett WC, et al. (1985) Reproducibility and validity of a semiquantitative food frequency questionnaire. Am J Epidemiol 122(1):51-65.

45. Lowe R, Morris T (2013) Report on the 2 nd annual Infinium Humanmethylation450 array workshop: 15 April 2013 QMUL, London, UK. Epigenetics 8(10):1123-1124.

46. Sandoval J, et al. (2011) Validation of a DNA methylation microarray for $450,000 \mathrm{CpG}$ sites in the human genome. Epigenetics 6(6):692-702.

47. Aryee MJ, et al. (2014) Minfi: A flexible and comprehensive Bioconductor package for the analysis of Infinium DNA methylation microarrays. Bioinformatics 30(10):1363-1369.

48. Pidsley $R$, et al. (2013) A data-driven approach to preprocessing Illumina $450 \mathrm{~K}$ methylation array data. BMC Genomics 14(1):293.

49. Bates D, Maechler M, Bolker B (2012) Ime4: Linear mixed-effects models using S4 classes. J Stat Software 67(1).

50. Kuznetsova A, Brockhoff PB, Christensen RHB (2013) ImerTest, R Package Version 2(6). Available at https://cran.r-project.org/web/packages/lmerTest/lmerTest.pdf. Accessed February 24, 2017

51. Peng C, et al. (2016) Particulate air pollution and fasting blood glucose in nondiabetic individuals: Associations and epigenetic mediation in the Normative Aging Study, 2000-2011. Environ Health Perspect 124(11):1715-1721.

52. Bind M-AC, Vanderweele TJ, Coull BA, Schwartz JD (2016) Causal mediation analysis for longitudinal data with exogenous exposure. Biostatistics 17(1):122-134. 